\title{
Combination of the Kinect with Virtual Reality in Balance Training for the Elderly
}

\author{
Wei-Min Hsieh ${ }^{1,2}$, Chih-Chen Chen ${ }^{3}$, Shih-Chuan Wang ${ }^{4}$, Yu-Luen Chen ${ }^{4,5 *}$, \\ Yuh-Shyan Hwang ${ }^{6}$, Jin-Shin Lai ${ }^{7}$ \\ ${ }^{1}$ Graduate Institute of Computer and Communication Engineering, National Taipei University \\ of Technology, Taipei, Chinese Taipei \\ ${ }^{2}$ Department of Electronic Engineering, Hwa Hsia Institute of Technology, Taipei, Chinese Taipei \\ ${ }^{3}$ Management Information System, Hwa Hsia Institute of Technology, Taipei, Chinese Taipei \\ ${ }^{4}$ Department of Computer Science, National Taipei University of Education, Taipei, Chinese Taipei \\ ${ }^{5}$ Department of Information Management, St. Mary’s Medicine, Nursing and Management College, Yilan, Chinese Taipei \\ ${ }^{6}$ Department of Electronic Engineering \& Institute of Computer and Communication, \\ National Taipei University of Technology, Taipei, Chinese Taipei \\ ${ }^{7}$ Department of Physical Medicine and Rehabilitation, National Taiwan University Hospital, \\ Taipei, Chinese Taipei \\ Email: *allen@tea.ntue.edu.tw
}

Received May 2013

\begin{abstract}
Daily life movements such as standing, walking, and jumping need balance ability to achieve. Good balance control is closely related to the body stability and its development. According to medical research, people showing dizziness after taking drugs may have their balance ability substantially affected, and are more likely to fall, especially true to the elderly. This study proposes the combination of Kinect with virtual reality to build an information platform of interactive scenarios, for practices and evaluation on balance ability. Based on the indicators of balance ability, this platform sets out various training activities to improve the balance ability, making the supposedly boring process fun and vivid for a much better training purposes. Also, according to literatures, training with gaming patterns results in $30 \%$ reduction of falls. What's more, this type of training can have the data, like time of use, scores, and joint postures, recorded and sent through network to databases on remote computer servers. The data collected from this platform can be sorted and analyzed, and the results can then be used to evaluate the performance of the balance training, and referenced for follow-up planning in the future.
\end{abstract}

Keywords: Balance; Kinect; Fall; Virtual Reality

\section{Introduction}

Balancing means the ability to maintain stability in all kinds of movements and postures. Most human movements rely on the foundation of balance, and keeping body balanced is critical to human movements. Body movements are pulled by gravity; a movement can be done only after the balancing point is achieved. Although normal people have decent balance ability to deal with daily needs, dizziness caused by diseases or drugs-especially taking multiple drugs, such as sleeping pills and tranquilizers, can bring about fall incidents.

Bureau of Health Promotion, Department of Health, points out in its Elderly Health Promotion Plan (20092012) that falling is one of the fetal causes of the elderly illness and death (51.0\%). Elderly people over the age of

*Corresponding author.
65 are the group with highest risk of fall death. People prone to fall are handicapped in activities of daily living (ADL) and instrumental activities of daily living (IADL). Researches also point out that $42.3 \%$ of the elderly people who suffer fall injuries need hospitalization, and $37.0 \%$ of them need to stay at nursing homes after the clinic therapies. Also, statistics show that in 2008 there are about 125,000 patients hospitalized for fall injuries. All the evidences tell the truth that aging does make substantial impact on the ability of balance control [1]. This study proposes the combination of Kinect with virtual reality to build an information platform of interactive scenarios, for practices and evaluation on balance ability. Based on the indicators of balance ability, this platform sets out various training activities to improve the balance ability, making the supposedly boring process fun and vivid for a much better training purpose. 
In his studies on older people's falling, Robbin et al. (1989) argues that abnormal gaits are in connection with the falls [2].

Daubney et al. (1999) studied 50 elderly people over the age of 65 , and analyzed the relationship between their lower limb muscle strengths and balances, and the results showed that the ones who fell without obvious reasons obviously had less balance ability than the ones who never fell, and the lower limb muscle strength was closely related to the balance ability; therefore, the muscle strength measurement index provides a very high prediction performance [3].

Robertson et al. (2002)'s research for a community showed that the elderly and people who fell before could go through exercise training to reduce the incidents of fall and alleviate fall injuries as well [4]. Ledin et al. (1990)'s research also showed that the elderly undertaking a period of time of balance training could have their balance ability improved to prevent fall and bone fracture [5].

In his study, Belgen et al. (2006) argues that individual body's balance ability is related to the probability of fall incidence, and people who suffer more fall incidents have less balance abilities [6].

D. Taylor et al. (2004) point out certain programmes of exercise such as the Falls Management Exercise (FaME) and Otago Exercise (OEP) programmes are effective in returning falls patients to normal functional movement and can reduce the risk of falling by as much as $30 \%$ [7].

R. Y. Wang et al. point out in their research projects (NSC100-2314-B010-022-MY2): falls among the elderly often lead to injury and thus lead to other complications. Exercise can prevent falls in older people. Previous studies also suggested integrated exercise training program was better than a single exercise. For healthy older adults, combining virtual reality (VR) with integrated exercise training program can enhance their motivation [8].

Y. R. Yang et al. point out in their research projects (NSC100-2314-B010-021-MY2): Exercise has beneficial physiologic effects in older adults, including effects on strength, aerobic capacity, and balance. These effects can reduce the risk and rate of falling among older adults. To effectively improve physical activities and to reduce risk of fall, appropriate exercise programs for specific fallers are needed [9].

\section{Material and Method}

Figure 1 is an overall schematic illustration, where Unity3D and Windows SDK software tools in combination with Kinect sensors are used to show body movements on a notebook computer at the other side.

Figure 2 shows the system structure of this study,

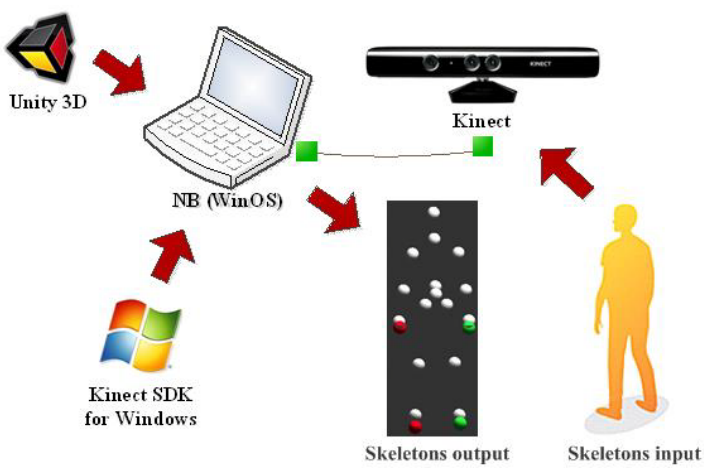

Figure 1. Overall schematic diagram.

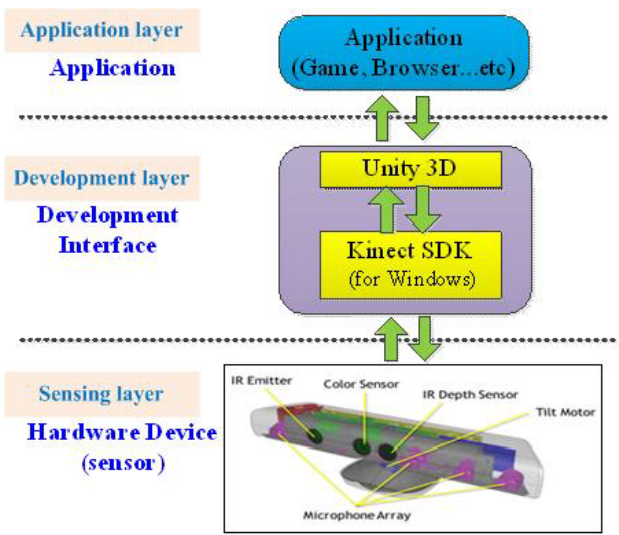

Figure 2. System structure diagram.

where the development process includes the hardware connections, signals receiving and analyses, movement identifying, etc. The hardware used is Kinect (for Windows) developed by Microsoft. The software application is developed with the Microsoft officially released Windows SDK software tool along with Unity3D, a 3Denabled game development software tool. The Windows SDK tool includes all the needed documents and API libraries and header files, while Unity3D is a comprehensive, 3D-game and 3D interactive tool for creativity development.

Kinect is used to detect human skeleton, and the detected 3D in-depth images are converted to the skeleton tracing system, which is capable of tracing up to 20 skeleton joints of the human body. The coordinates of the detected skeleton joints are then reflected in the 3D scenes developed with Windows SDK programs using Unity3D's libraries to visualize the body movements and present the visual reality simulation.

This study mainly focuses on fall prevention, and the proposed training and test items are designed by taking references to the Four Square Step Test (FSST) method often used for examining balance ability; one of the designed activities is using the hand or foot or the combination of foot and hand to touch the random balls for the balance training or test. The lead preparation for the 
training and test activities is shown in Figure 3, where the medical personnel needs to pre-select the difficulty level, applicable module, evaluation method, and suitable postures.

The degree of difficulty of the stretching movements is defined by the participant's skeleton. To take into account safety during the activities, Figure 4 demonstrates the scope of the stretching movements, where the $50 \%$, $75 \%$, and $100 \%$ degree between SHOULDER CENTER and WRIST are easy, medium, and difficult stretching, respectively, which are corresponded with the random balls appearing at specific spots designed by the software programs.

The program needs to be initialized before the training or test starts, i.e. benchmarking the participant's position and skeleton, followed by limb stretching warm-up before entering the training procedure. Figure 5 is a block diagram showing the initialization, warm-up and training. The activities are conducted in a game-like manner in order to increase the participant's interest in doing the activities. This system also provides the training data that can be used for evaluation on the training effectiveness.

Figure 6 is a block diagram of the test, which requires the lead process and initialization before being commenced. The result can be based on fixed time to calculate the scores or on fixed scores to calculate the time.

\section{Results}

This study takes an empirical test on Mr. A of 67 years of age, who is $178 \mathrm{~cm}$ high, and weighted $83 \mathrm{~kg}$. With the developed virtual-reality training program, the tested male uses his hand or foot to touch the random balls, and the time used to finish the given number of touches on the balls is measured for the balance training.

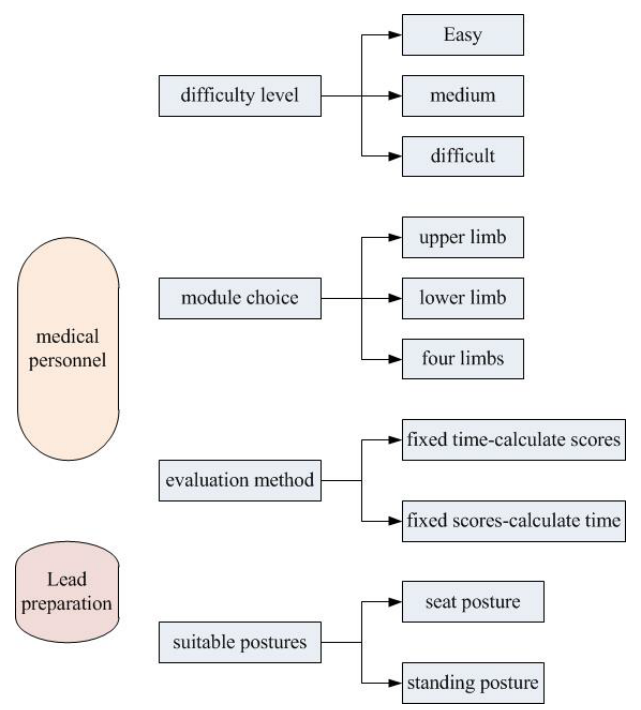

Figure 3. Lead preparation for the training and test activities.

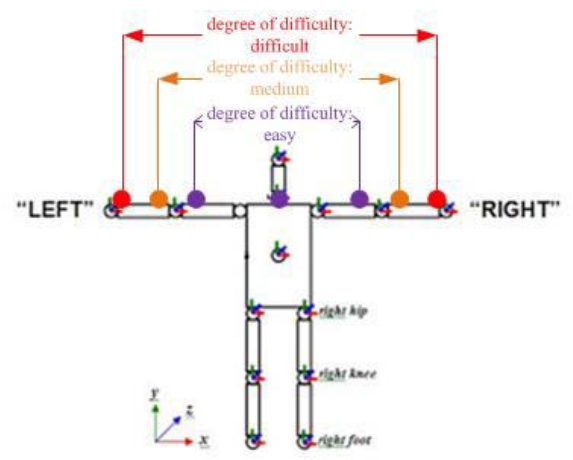

Figure 4. The scope of the stretching movements.

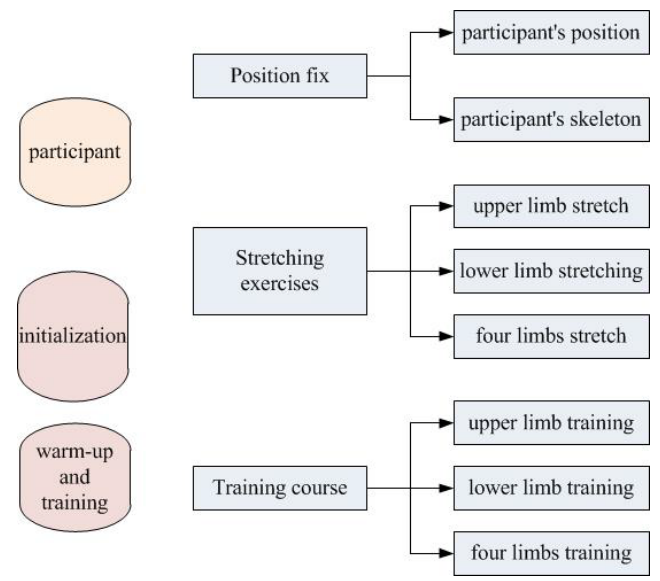

Figure 5. The initialization, warm-up and training block diagram.

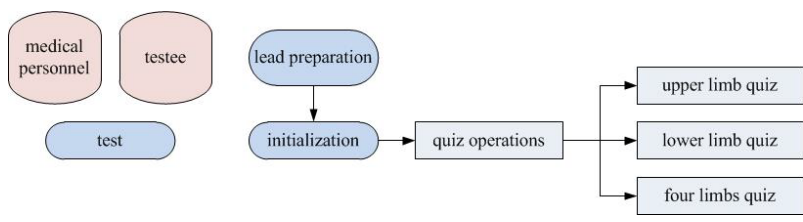

Figure 6. Test block diagram.

Figure 7 shows the screen of the hand touching the ball, where the ball appears on the upper right corner, and the tester scores with his right hand touching the ball. On this screen, (a) shows the right hand has not yet touched the ball, and (b) shows the right hand are touching the ball.

A training program was set as a six-week session, and the difficulty level was easy palm or foot exercise. Each of the exercises was given 3 games, with a 2-minute interval between games. There was a 5-minute break before switching to a different touch method. In each game, the random ball showed up 5 times, and each of which was to be touched by a specific limb. The time used for the games was calculated. And the times used were averaged by each different touch method. Table 1 lists the results from the elderly Mr. A's training, which shows the times used for these two training methods, and the 


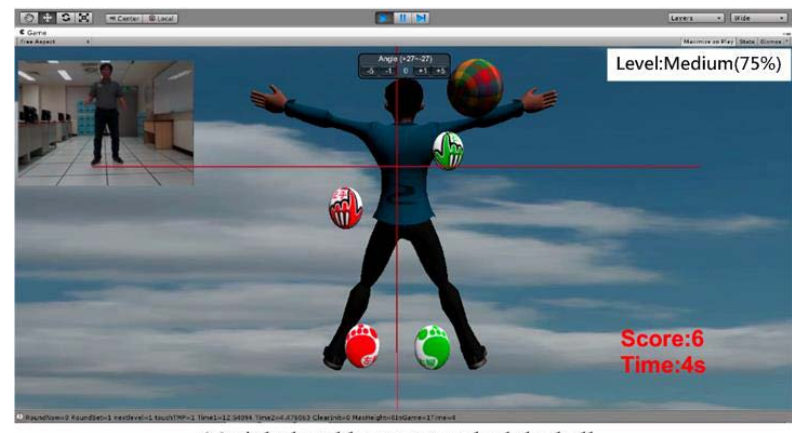

(a) right hand has not touched the ball

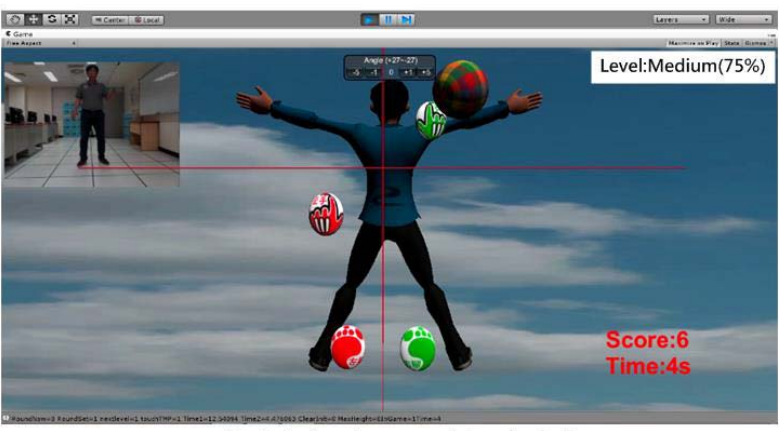

(b) right hand are touching the ball

Table 7. The screen of the hand touching the ball.

Table 1. Lists of the training results.

\begin{tabular}{ccccccc} 
Week/Limb & Week 1 & Week 2 & Week 3 & Week 4 & Week 5 & Week 6 \\
\hline Hand & $35 \mathrm{sec}$ & $34 \mathrm{sec}$ & $32 \mathrm{sec}$ & $32 \mathrm{sec}$ & $31 \mathrm{sec}$ & $29 \mathrm{sec}$ \\
Foot & $37 \mathrm{sec}$ & $36 \mathrm{sec}$ & $36 \mathrm{sec}$ & $35 \mathrm{sec}$ & $33 \mathrm{sec}$ & $32 \mathrm{sec}$
\end{tabular}

results from adjacent weeks show either improved or unchanged. Also, Mr. A's training records of the times used by his palm and foot show that his foot reaction is weaker than his palm. An interview with elderly Mr. A reveals that his foot received medical treatment because of sports injuries, and that's why his foot is weaker in reaction. Figure 8 shows the averaged weekly time used for his hand or foot to touch the random balls.

\section{Conclusion}

This study proposes the combination of Kinect with virtual reality to build an information platform of interactive scenarios, for practices and evaluation on balance ability. A clinical assessment test was given to another elderly person, and it was also proved that after the 6-week training, the tester obviously made progress in reactions. From the training results of the participants, we know that the information platform developed for enhancing the balance ability is in line with the practical needs, and the fun and interesting game-like exercises it introduces are much helpful to the improvement of balance ability, and certainly to the prevention of falling. In the future, more tests will be given on the elderly in order to collect

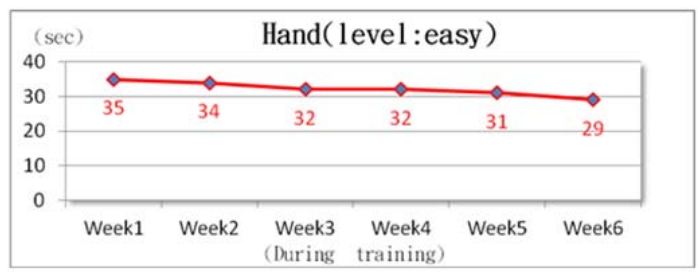

(a)

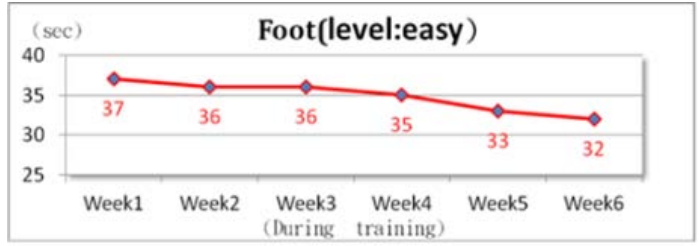

(b)

Figure 8. The averaged weekly time used for hand or foot. (a) theaveraged weekly time of hand; (b) the averaged weekly time of foot.

more effective and comprehensive quantitative data for the system's fine tuning and modifications.

\section{Acknowledgements}

The authors wish to thank the National Science Council (NSC) in Taiwan (Grant Number: NSC 101-2221-E-146 -001-, NSC101-2221-E-152-001-, NSC101-2627-E-002006-) for support this research.

\section{REFERENCES}

[1] Bureau of Health Promotion, Department of Health, Chinese Taipei.

http://www.bhp.doh.gov.tw/bhpnet/portal/PressShow.asp $\mathrm{x} ? \mathrm{No}=200911060002$

[2] A. S. Robbin and L. Z. Rubenstein, "Predictors of Fall among Elderly People,” Archives of Internal Medicine, Vol. 49, 1989, pp. 1628-1633.

[3] M. E. Daubney and E. G. Culham, "Lower-Extremity Muscle Fore and Balance Performance in Adults Aged 65 Years and Older”, Physical Therapy, Vol. 79, No. 12, 1999, pp. 1177-1185.

[4] M. C. Robertson, A. J. Campbell, M. M. Gardner and N. Devlin, "Preventing Injuries in Older People by Preventing Falls: A Meta-Analysis of Individual-Level Data," Journal of the American Geriatrics Society, Vol. 50, 2002, pp. 905-911.

[5] T. Ledin, A. C. Kronhed, C. Moller, M. Moller, L. M. Odkvist and B. Olsson, "Effects of Balance Training in Elderly Evaluated by Clinical Tests and Dynamic Petrography," Journal of Vestibular Research, Vol. 1, No. 2, 1990, pp. 129-138.

[6] B. Belgen, M. Beninato, P. E. Sullivan and K. Narielwalla, "The Association of Balance Capacity and Falls Self- efficacy with History of Falling in Community-Dwelling People with Chronic Stroke,” The American Congress of Rehabilitation Medicine and the American Academy of 
Physical Medicine and Rehabilitation, Vol. 87, 2006, pp. 554-561.

[7] D. Taylor and C. Stretton, "The Otago Exercise Program, An Evidence-Based Approach to Falls Prevention for Older Adults Living in the Community," Journal of Primary Health Care, Vol. 31, No. 6, 2004.

[8] R. Y. Wang, "Virtual Reality-Enhanced Optimal Exercise
Training Model for Falls Prevention and Its Remote Applications in Elderly with Different Fall Risks," NSC 100-2314-B010-022-MY2, 2011.

[9] Y. R. Yang, "Optimized Exercise Model and Remote Training for Fall Prevention in Elderly (I),” NSC1002314-B010-021-MY2, 2011. 